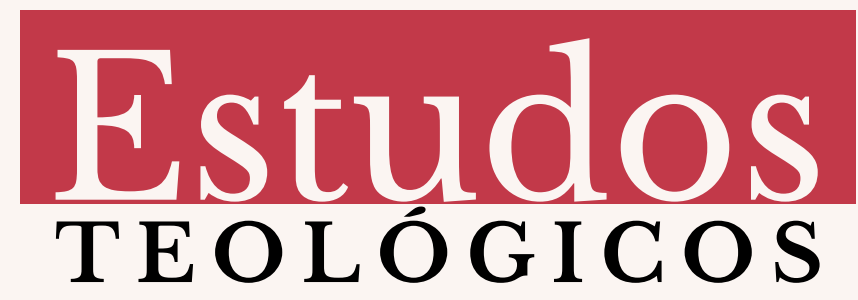

Programa de Pós-Graduação em Teologia da Faculdades EST

Lista dos participantes do conselho científico - pessoas avaliadoras dos textos - ad hoc

(em ordem alfabética)

\title{
Ano 2020
}

Adriano Sousa Lima (FABAPAR/UNINTER, Curitiba/PR, Brasil), Ana Rosa Cloclet da Silva (PUC de Campinas, Campinas/SP), André Luiz Boccato Almeida (PUCSP, São Paulo/SP, Brasil); André S. Musskopf (UFJF, Juiz de Fora/MG, Brasil), Arno Scheunemann (ULBRA, Canoas/RS), Carlos R. Caldas Filho (PUC-Minas, Belo Horizonte/MG), Carolina Bezerra de Souza (Faculdades EST, São Leopoldo/RS), Carolina Gual Silva (Universidade Estadual de Campinas, Campinas/SP), Celso Gabatz (Faculdades EST, São Leopoldo/RS, Brasil). César Lopes (FATIPI, São Paulo/SP, Brasil), Cesar Motta Rios (UFMG, Belo Horizonte/MG, Brasil); Claudete Beise Ulrich (FUV, Vitória/ES, Brasil), Claudio de Oliveira Ribeiro (PUC, Campinas/SP, Brasil), Clélia Peretti (PUCPR, Curitiba/PR, Brasil); Daniele Galindo (UFPEL, Pelotas/RS, Brasil), Darlei de Paula (PUC-RS, Porto Alegre/RS), David Mesquiati de Oliveira (FUV, Vitória/ES, Brasil); Diego Genu Klautau (Centro Universitário FEl, São Bernardo do Campo/SP),

Éder Beling (pesquisador independente), 


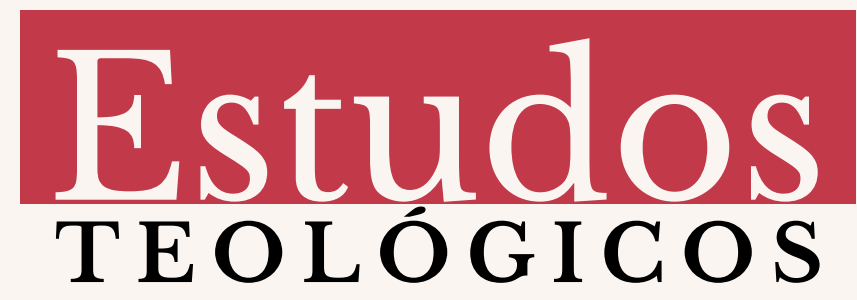

Programa de Pós-Graduação em Teologia da Faculdades EST

Edla Eggert (PUCRS, Porto Alegre/RS, Brasil),

Eduardo Gross (UFJF, Juiz de Fora/MG, Brasil),

Elias Wolff (PUCPR, Curitiba/ PR),

Etienne Higuet (Universidade do Estado do Pará, Belém/PA),

Euler Westphal (Universidade da Região de Joinville, Joinville/SC),

Evaldo L. Pauly (UNILASALLE, Canoas/RS),

Ezequiel Hanke (Faculdades EST, São Leopoldo/RS),

Fabrício Veliq (FAJE, Belo Horizonte/MG, Brasil),

Fernando Albano (Faculdade Refidim, Joinville/SC),

Flávio Schmitt (Faculdades EST, São Leopoldo/RS),

Flávio Senra (PUC Minas, Belo Horizonte/MG, Brasil),

Francisco de Assis dos Santos (FUV, Vitória/ES, Brasil),

Gerson Leite de Moraes (Universidade Presbiteriana Mackenzie, São

Paulo/SP),

Gilbraz Aragão (Universidade Católica de Pernambuco, Recife/PE, Brasil);

Clauco Barsalini (PUC-Campinas, Campinas/SP),

Heiko Grünwedel (Faculdades EST, São Leopoldo/RS, Brasil);

luri Andréas Reblin (Faculdades EST, São Leopoldo/RS, Brasil);

Ivoni Richter Reimer (PUC-Goiás Goiânia/CO, Brasil),

Jefferson Zeferino (PUCPR, Curitiba/ PR, Brasil);

Joe Marçal G. Santos (Universidade Federal de Sergipe, São Cristóvão/SE, Brasil);

Joel Haroldo Baade (UNIARP, Caçador/SC, Brasil),

José Mário Gonçalves (UFES/FUV, Vitória/ES),

Júlio Cézar Adam (Faculdades EST, São Leopoldo/RS, Brasil);

Jung Mo Sung (Universidade Metodista de São Paulo, São Bernardo do Campo/SP), 


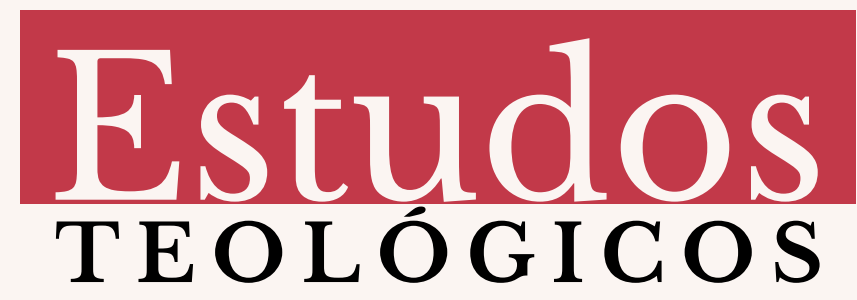

Programa de Pós-Graduação em Teologia da Faculdades EST

Kathlen Luana de Oliveira (IFRS, Osório/RS, Brasil);

Lauri Emilio Wirth (UMESP, São Bernardo do Campo/SP, Brasil),

Luiz Carlos Ramos (Universidade São Francisco, São Paulo/SP),

Manoel Freire (UERN, Açu/RN, Brasil);

Marcelo da Silva Carneiro (UMESP, São Bernardo do Campo/SP, Brasil),

Marcia Blasi (Faculdades EST, São Leopoldo/ RS),

Marcial Maçaneiro (PUCPR, Curitiba/PR, Brasil);

Martin Dietz (IECLB, São Leopoldo/RS, Brasil);

Mary Rute Gomes Esperandio (PUCPR, Curitiba/PR, Brasil);

Nilton Eliseu Herbes (Faculdades EST, São Leopoldo/RS),

Noli Bernardo Hahn (URI, Santo Ângelo/RS, Brasil),

Oneide Bobsin (Faculdades EST, São Leopoldo/RS, Brasil);

Osvaldo Ribeiro (FUV, Vitória/ES, Brasil);

Regiano Bregalda (Universidade de Passo Fundo, Passo Fundo/RS), Pedro Simões (Universidade Federal de Santa Catarina, Florianópolis/SC),

Renato Kirchner (PUC de Campinas, Campinas/SP),

Roberto Ervino Zwetsch (EST, São Leopoldo/RS, Brasil),

Roberto Hofmeister Pich (PUCRS, Porto Alegre/RS, Brasil);

Rodrigo Coppe Caldeira (PUC-Minas, Belo Horizonte/MG),

Rodrigo Portella (UFJF, Juiz de Fora/MC, Brasil),

Romeu Ruben Martini (IECLB, Porto Alegre/RS, Brasil),

Rosângela Angelin (Universidade Regional Integrada do Alto Uruguai e das Missões, Santo Ângelo/RS),

Rudolf von Sinner (PUCPR, Curitiba/PR, Brasil);

Scheila Janke (Universität Göttingen, Alemanha), 


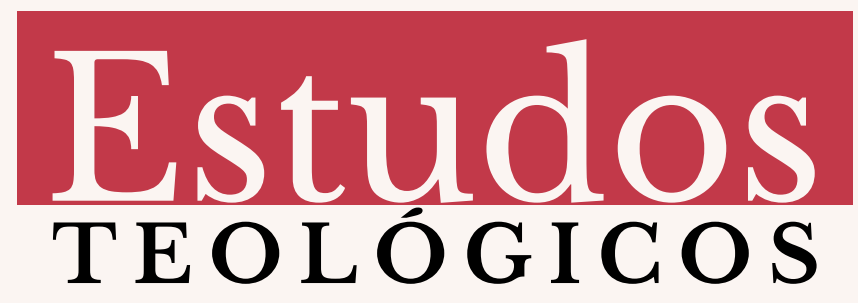

Programa de Pós-Graduação em Teologia da Faculdades EST

Sérgio Rogério Azevedo Junqueira (PUCPR, Curitiba/PR, Brasil); Thomas Heimann (ULBRA, Canoas/RS, Brasil), Uwe Wegner (Faculdades EST, São Leopoldo/RS, Brasil);

Valério C. Schaper (EST, São Leopoldo/RS, Brasil),

Vitor Barletta (PUC-Campinas, Campinas/SP),

Waldir Souza (PUCPR, Curitiba/PR),

Wilhelm Sell (IECLB, Porto Alegre/RS),

Wilhelm Wachholz (Faculdades EST, São Leopoldo/RS, Brasil); 